\title{
ON THE FOURIER COEFFICIENTS AND CONTINUITY OF FUNCTIONS OF CLASS $\mathscr{V}_{\Phi}^{*}$
}

\author{
ELAINE COHEN
}

\begin{abstract}
Let $f$ be a periodic function with a fractional intergral $f_{(r)}$ or fractional derivative $f^{(r)}$ of class $\mathscr{V}_{\Phi}{ }^{*}$. For a class of Young's functions, this paper presents necessary and sufficient conditions for continuity of $f_{(r)}$ or $f^{(r)}$ in terms of the orders of magnitude of the partial sums of the absolute values of the Fourier coefficients of $f$. Sufficient conditions are presented for another class of Young's functions. Also, results on the order of magnitude of the Fourier coefficients of $f$ are derived.
\end{abstract}

1. Introduction. For real functions $f$ of period $2 \pi$ with $r$-th fractional derivative, $r \geqq 0$, of bounded $p$-variation, Golubov [4] has obtained conditions for continuity of the $r$-th derivative in terms of the moduli of the Fourier coefficients. We consider the analogous problem for functions whose fractional derivatives are of $\Phi$-bounded variation, and also obtain estimates on the order of magnitude of the Fourier coefficients. Below, we shall briefly define the terms used and state some elementary properties. For a short summary of the properties of these terms see Cohen [2]. More complete discussions are in Zygmund [11], Krasnosel'skii and Rutickii [5], L. C. Young [10], E. R. Love [6], and Musielak and Orlicz [7].

We define an $N$-function or Young's function to be any convex, strictly increasing function $\Phi$ such that $\lim _{u \rightarrow \infty} \Phi(u) / u=\infty$ and $\lim _{u \rightarrow 0}$ $\Phi(u) / u=0$. Furthermore, an $N$-function $\Phi$ satisfies the $\Delta^{\prime}$ condition (or $\Phi$ is $\Delta^{\prime}$ ) for (small; large) values if there exists $c>0$ (and $u_{0}>c$ ) such that (for $\left.|x|,|y| \leqq u_{0} ;|x|,|y| \geqq u_{0}\right)$

$$
\Phi(x y) \leqq c \Phi(x) \Phi(y) .
$$

If $\Phi$ is $\Delta^{\prime}$ for small values, say $|u| \leqq u_{0}$, then we can replace $u_{0}$ by any $w>u_{0}$ but the $\Delta^{\prime}$ constant $c$ increases unboundedly with $w$ unless $\Phi$ is $\Delta^{\prime}$ for all values.

For an $N$-function $\Phi$, define

$$
V_{\Phi}(f ; I)=\sup _{Q} \sum \Phi\left(f\left(x_{i}\right)-f\left(x_{i-1}\right)\right)
$$

where the supremum is taken over all partitions $Q$ of the interval $I$. We call $V_{\Phi}(f ; I)$ the $\Phi$-varation of $f$ on $I . V_{\Phi}(f)$ and "the $\Phi$-variation of $f$ " is used when the interval is of length $2 \pi$.

Received by the editors on February 7, 1977.

Copyright $\odot 1979$ Rocky Mountain Mathematical Consortium 
The class of functions of $\Phi$-bounded variation $(\Phi B V)$ is

$$
\mathscr{V}_{\Phi}(I)=\left\{f \in L^{1}(I): V_{\Phi}(f ; I)<\infty\right\} .
$$

Closely related definitions are

$$
V_{\Phi}^{(\delta)}(f ; I)=\sup _{|Q| \leqq \delta} \sum \Phi\left(f\left(x_{i}\right)-f\left(x_{i-1}\right)\right)
$$

and

$$
V_{\Phi}^{*}(f ; I)=\lim _{\delta \rightarrow 0^{+}} V_{\Phi}^{(\delta)}(f ; I),
$$

where $|Q|$ denoted the mesh of the partition $Q$, and

$$
\mathscr{Y}_{\Phi}{ }^{*}(I)=\left\{f:(k f) \in \mathcal{Y}^{\prime}{ }_{\Phi}(I) \text { for some real } k \neq 0\right\} .
$$

Functions $f$ of $\Phi$ bounded variation can have simple discontinuities only. We shall assume that they are normalized so that

$$
f(x)=\frac{1}{2}(f(x+)+f(x-)) .
$$

It can be shown that a necessary and sufficient condition for $V_{\Phi}(f ; I)$ to be finite is that $V_{\Phi}{ }^{*}(f ; I)$ is finite. Later proofs will use the two definitions interchangeably.

Suppose $f$ is of period $2 \pi, f$ has mean value zero $\left(\int f=0\right)$ and

$$
f(x) \sim \sum^{\prime} c_{n} e^{i n x}=\sum_{n \neq 0} c_{n} e^{i n x}
$$

For $r>0$ set

$$
(\text { in })^{-r}=|n|^{-r} \exp \left(-\frac{1}{2} i \pi r \text { sgn } n\right),
$$

and define

$$
D^{(r)}(t)=\Sigma^{\prime}(\text { in })^{-r} e^{i n t} .
$$

If $r$ is an interger $D^{(r)}$ is a polynomial and

$$
\frac{1}{2} \pi \int f(t) D^{(r)}(x-t) d t
$$

is an $r$-th order primitive of $f$. For any real $r>0$ we define the fractional integral of order $r$ of $f$ :

$$
f_{(r)}(x)=\int f(t) D^{(r)}(x-t) d t .
$$

It can be shown that $f_{(r)}(x)$ exists almost everywhere, is integrable, and

$$
f_{(r)}(x) \sim \sum^{\prime} \frac{c_{n} e^{i n x}}{(i n)^{r}}
$$


We say that $f^{(r)}$ is the fractional derivative of $f$ of order $r$ if

$$
f(x)=\int D^{(r)}(x-t) f^{(r)}(t) d t \quad \text { a.e., }
$$

i.e., $f$ is a fractional integral of $f^{(r)}$ of order $r$.

We let

$$
\mathscr{Y}_{\Phi}^{\prime}(r)=\left\{f: f^{(r)} \text { exists and } f^{(r)} \in V_{\Phi}\right\} .
$$

Define $W$ as the set of $2 \pi$ periodic functions with no discontinuities of the second kind, and such that

$$
\min \{g(t-), g(t+)\} \leqq g(t) \leqq \max \{g(t-), g(t+)\} .
$$

Then $V_{\Phi} \subseteq W$ for all $N$-functions $\Phi$.

2. Suppose that $g \in \mathscr{Y}_{\Phi}$ for any $N$-function $\Phi$ and that

$$
g(t) \sim \sum_{k=1}^{\infty} \alpha_{k} \cos k t+\beta_{k} \sin k t
$$

and

$$
\rho_{k}(g)=\left(\alpha_{k}{ }^{2}+\beta_{k}^{2}\right)^{1 / 2} .
$$

Consider the following conditions on a sequence of non-negative real numbers $\left\{\rho_{k}\right\}$ :

$$
\begin{aligned}
& \sum_{k=1}^{n} \quad k^{2}{\rho_{k}}^{2}=\mathrm{o}(n) \\
& \sum_{k=1}^{n} \quad k \rho_{k}=\mathrm{o}(n) \\
& \sum_{k=1}^{n} \quad \rho_{k}=\mathrm{o}(\log n) \\
& \sum_{k=n}^{\infty} \quad \rho_{k}{ }^{2}=\mathrm{o}(l / n) .
\end{aligned}
$$

The following result is well known [3].

Lemma 2.1. Conditions (I)-(IV) are related as follows (IV) $\Rightarrow(\mathrm{I}) \Rightarrow$ (II) $\Rightarrow$ (III).

In the same paper Golubov has proven the following result which we shall require. 
TheOREM 2.2. If $g \in W$, and $\left\{\rho_{n}\right\}$ is as in equation (2.2), each of the conditions (I)-(IV) is sufficient for $g$ to be continuous; however, there is no condition on $\left\{\rho_{k}\right\}$ which is necessary and sufficient.

For a smaller class of functions we make this result somewhat more precise in the following theorem.

Theorem 2.3. Let $\Phi$ be an $N$-function and let $g \in \mathscr{V}_{\Phi}{ }^{*}$, and equations (2.1) and (2.2) hold. We have

(a) if $\lim _{u \rightarrow 0} u^{2} / \Phi(u)=0$, each of the conditions (I)-(IV) is necessary and sufficient for $g$ to be continuous,

(b) if $\lim \inf _{u \rightarrow 0} u^{2} / \Phi(u) \neq 0$, each of the conditions (I)-(IV) is still sufficient, but there is no condition on $\left\{\rho_{k}\right\}$ which is necessary and sufficient.

Proof. (a) Since $\mathscr{V}_{\Phi}{ }^{*} \subset W$ for all $I$, by Theorem 2.2 , we have that each condition is sufficient for the continuity of $g \in \mathscr{V}_{\Phi}{ }^{*}$. Now, suppose $\lim _{u \rightarrow 0} u^{2} / \Phi(u)=0, g \in \mathscr{V}_{\Phi}{ }^{*}$ and $g$ is continuous.

$$
g(t+h)-g(t-h) \sim 2 \sum_{1}^{\infty}\left(-\alpha_{k} \sin k t+\beta_{k} \cos k t\right) \sin k h .
$$

Cohen [2] has shown that for $f \in \mathscr{V}_{\Phi}{ }^{*}$ there exists $b \neq 0$ such that

$$
\sup _{|h| \leqq \delta} \int_{0}^{2 \pi} \Phi(b f(x \mathrm{th})-b f(x)) d x \leqq 3 \delta V_{\Phi}^{(2 \delta)}(b f) .
$$

Now $\lim _{u \rightarrow 0} u^{2} / \Phi(u)=0$ implies $\mathscr{V}_{\Phi} \subseteq \mathscr{V}_{2}$, so using Parseval's formula and equation 2.3 , for some $b \neq 0$,

$$
\begin{aligned}
& 4 b^{2} \sum_{k=1}^{\infty} \rho_{k}^{2} \sin k h \\
& =\frac{1}{\pi} \int_{0}^{2 \pi} b^{2}|g(t+h)-g(t-h)|^{2} d t \\
& \leqq \frac{1}{\pi} \sup \left\{\frac{\left(b g(x+t)-b(g(x-t))^{2}\right.}{\Phi(b g(x+t)-b g(x-t))}: \mathrm{x} \in[0,2 \pi] ;|t| \leqq h\right\} \\
& \quad \cdot \int_{0}^{2 \pi} \Phi(b g(t+h)-b g(t-h)) d t \\
& =\mathrm{o}(1) \mathrm{o}(h)=\mathrm{o}(h) .
\end{aligned}
$$

From Wiener [9] and above, 


$$
\frac{1}{4} \limsup _{n \rightarrow \infty}\left(n \sum_{k=n}^{\infty}{\rho_{k}}^{2}\right) \leqq \underset{n \rightarrow \infty}{\limsup }\left(n \sum_{k=1}^{\infty}{\rho_{k}}^{2} \sin \frac{k \pi}{n}\right)=\mathrm{o}(1)
$$

so condition (IV) is satisfied, and by Lemma 2.1, conditions (I)-(III) are also satisfied.

(b) Consider the functions

$$
f_{1}(t)=\sum_{k=1}^{\infty} k^{-1} \sin k t=\left\{\begin{array}{cc}
\frac{\pi-t}{2} & 0<t<2 \pi \\
0 & t=0
\end{array}\right.
$$

with discontinuity at $t=0$, and

$$
f_{2}(t)=\sum_{k=1}^{\infty} k^{-1} \sin k(t+\log k)
$$

Both series converge for all $t, f_{1}(0)=0, f_{1}(0+)=\frac{\pi}{2}, f_{1}(0-)=-\frac{\pi}{2}$, and $f \in \mathscr{V}_{1}$, while $f_{2} \in \operatorname{Lip} 1 / 2[11$, v. I, p. 197] and hence is continuous. We also have $\rho_{k}\left(f_{1}\right)=\rho_{k}\left(f_{2}\right)$ for all $k$.

Now if $\lim \inf _{u \rightarrow 0} u^{2} / \Phi(u) \neq 0$, then there exists $u_{0}>0$ and $A>0$ such that for $0 \leqq u \leqq u_{0}, \Phi(u) \leqq A u^{2}$.

Thus, $\mathscr{V}_{2}{ }^{*} \subseteq \mathscr{V}_{\Phi}{ }^{*}$ and since Lip $1 / 2 \subseteq \mathscr{V}_{2}$,

$$
f_{2} \in \mathscr{V}_{\Phi}^{*} \text {. }
$$

Since $\mathscr{V}_{1} \subseteq \mathscr{V}_{\Phi}$, we have $f_{1} \in \mathscr{V}_{\Phi}$. Thus, no condition on $\left\{\rho_{k}\right\}$ can be necessary and sufficient for the continuity of a function in this case.

The above theorem admits several generalizations.

Corollary 2.4. Let $f^{(r)} \in \mathscr{V}_{\Phi}{ }^{*}, r \geqq 0$. Then

(a) if $\lim _{u \rightarrow 0} u^{2} / \Phi(u)=0$ each of the following conditions is equivalent to the continuity of $f^{(r)}\left(\rho_{k}=\rho_{k}(f)\right.$ :

$$
\sum_{k=1}^{n} k^{2 r+2}{\rho_{k}}^{2}=\mathrm{o}(n)
$$

$$
\sum_{k=1}^{n} k^{r+1} \rho_{k}=\mathrm{o}(n)
$$

$$
\sum_{k=1}^{n} k^{r} \rho_{k}=\mathrm{o}(\log n)
$$

$$
\sum_{k=n}^{\infty} k^{2 r} \rho_{k}^{2}=\mathrm{o}(1 / n)
$$


(b) If lim $\inf _{u \rightarrow 0} u^{2} / \Phi(u) \neq 0$ then each of the conditions (I)-(IV) is still sufficient, but there is no condition on $\left\{\rho_{k}\right\}$ which is necessary and sufficient.

Proof. Since $f(t)=\int D^{(r)}(x-t) f^{(r)}(t) d t$, if $f^{(r)} \sim \Sigma \alpha_{k} \cos k x+$ $\beta_{k} \sin k x$ then

$$
\begin{aligned}
f & \sim \sum n^{-r} \cos k x\left(\alpha_{n} \cos \frac{1}{2} \pi r-\beta_{n} \sin \frac{1}{2} \pi r\right) \\
& +n^{-r} \sin k x\left(\beta_{n} \cos \frac{1}{2} \pi r+\alpha_{n} \sin \frac{1}{2} \pi r\right)
\end{aligned}
$$

and

$$
\begin{aligned}
\rho_{k}(f)= & n^{-r}\left[\left(\alpha_{n} \cos \frac{1}{2} \pi r-\beta_{n} \sin \frac{1}{2} \pi r\right)^{2}\right. \\
& \left.+\left(\beta_{n} \cos \frac{1}{2} \pi r+\alpha_{n} \sin \frac{1}{2} \pi r\right)^{2}\right]^{1 / 2} \\
= & n^{-r}\left[\alpha_{n}{ }^{2}+\beta_{n}{ }^{2}\right]^{1 / 2} \\
= & n^{-r} \rho_{k}\left(f^{(r)}\right) .
\end{aligned}
$$

So applying Theorem 2.3 to

$$
\rho_{k}\left(f^{(r)}\right)=n^{r} \rho_{k}(f)
$$

the result follows.

Corollary 2.5. Suppose $f_{(r)} \in \mathscr{V}_{\Phi}^{*}, 0<r<1$.

(a) If $\lim _{u \rightarrow 0} u^{2} / \Phi(u)=0$, then each of the following is equivalent to the continuity of $f_{(r)}$ :

$$
\begin{aligned}
& \sum_{k=1}^{n} k^{2-2 r}{\rho_{k}}^{2}=o(n) \\
& \sum_{k=1}^{n} k^{1-r} \rho_{k}=o(n)
\end{aligned}
$$

$$
\begin{aligned}
& \sum_{k=1}^{n} k^{-r} \rho_{k}=\mathrm{o}(\log n) \\
& \sum_{k=n}^{\infty} k^{-2 r}{\rho_{k}}^{2}=\mathrm{o}(1 / n) .
\end{aligned}
$$

(b) if $\lim \inf _{u \rightarrow 0} u^{2} / \Phi(u) \neq 0$, then each of the conditions (I)-(IV) is still sufficient, but there is no condition on $\left\{\rho_{k}\right\}$ which is necessary and sufficient. 
Proof. $k^{-r} \rho_{k}(f)=\rho_{k}\left(f_{(r)}\right)$.

THEOREM 2.6. If $f \in \mathscr{V}_{\Phi}^{*}$ and if $\Phi, \Psi$ are $N$-functions such that $\lim _{x \rightarrow 0} \Psi(x) / \Phi(x)=0$ then $f$ is continuous iff, for some $b \neq 0$, $\int_{0}^{2 \pi} \Psi(b(f(x+h)-f(x))) d x=o(|h|)$.

Proof. Let $f \in \mathscr{V}_{\Phi}{ }^{*}$ be continuous, $\Psi$ as above. By equation (2.3),

$$
\begin{aligned}
& \int_{0}^{2 \pi} \Psi(b(f(t+h)-f(t))) \\
& \leqq \sup \left\{\frac{\Psi(b f(t+u)-b f(t))}{\Phi(b f(t+u)-b f(t))}: t \in[0,2 \pi],|u| \leqq h\right\} \\
& \cdot \int_{0}^{2 \pi} \Phi(b(f(t+h)-f(t))) d t \\
&= o(1) \cdot \sigma(|h|) \\
&= o(|h|) .
\end{aligned}
$$

Conversely, suppose $f$ has jump $d>0$ at $x_{0}$. For $h$ sufficiently small, then

$$
|f(x+h)-f(x)|>d / 2
$$

in an interval of length $|h|$. Thus

$$
\int_{0}^{2 \pi} \Psi(b(f(x+h)-f(x))) d x>|h| \Psi\left(\frac{d b}{2}\right) \neq \mathrm{o}(|h|) .
$$

Therefore, $\int_{0}^{2 \pi} \Psi(b(f(x+h)-f(x))) d x=o(|h|)$ implies $f$ is continuous.

DEFinition. Let $\omega_{\Phi}(1 ; f ; \delta)=V_{F}^{(\delta)}(f)$ and then define

$$
\begin{aligned}
\omega_{\Phi}(k ; f ; \delta) & =\sup _{|h| \leqq \delta} \omega_{\Phi}\left(1 ; \Delta_{h}^{k-1} f ;|h|\right) \\
& =\sup _{|h| \leqq \delta} V_{\Phi}^{(|h|)}\left(\Delta_{h}^{k-1} f\right),
\end{aligned}
$$

where

$$
\begin{aligned}
\Delta_{h}{ }^{k} f(t) & =\sum_{v=0}^{k}(-1)^{v}\left(\begin{array}{c}
k \\
v
\end{array}\right) f(t+v h) \\
& =\Delta_{h}^{k-1} f(t+h)-\Delta_{h}^{k-1} f(t)
\end{aligned}
$$

and $k=2,3, \cdots$. 
Lemma 2.7. Suppose $\Phi$ is $\Delta^{\prime}$ and $f \in \mathscr{V}_{\Phi}^{(r)}, 0 \leqq r$ and $1 \leqq k$. Then

$$
\begin{aligned}
\max \left(\left|a_{n}\right|,\left|b_{n}\right|\right) \leqq & \frac{1}{n^{r} \Phi^{-1}(n)} \cdot \frac{1}{\Phi^{-1}\left(\frac{1}{\omega_{\Phi}\left(k, f^{(r)}, \pi / n\right)}\right)} \\
& \cdot \frac{1}{2^{k-1} \Phi-\left(1 / c_{\Phi}^{3} \pi\right) \Phi^{*}\left(\frac{1}{2} \pi\right) \pi} .
\end{aligned}
$$

Proof. Let $g \in \mathscr{V}_{\boldsymbol{\Phi}}$,

$$
g(x) \sim \sum \alpha_{n} \cos n x+\beta_{n} \sin n x .
$$

Then

$$
2^{k} \alpha_{n}=\frac{1}{\pi} \int_{0}^{2 \pi}\left\{\Delta_{\pi / n}^{k} g(t)\right\} \cos n t d t
$$

Using Hölders inequality,

$$
2^{k}\left|\alpha_{n}\right| \leqq\left\|\Delta_{\pi / n}^{k} g(t)\right\|_{\Phi}^{\prime}\left\|\frac{1}{\pi} \cos n t\right\| \Phi_{\Phi^{*}} .
$$

Now

$$
\begin{aligned}
& \left\|\Delta_{\pi / n}^{k} g(t)\right\|_{\Phi}^{\prime}=\left\|\Delta_{\pi / n}^{k-1} g(t+\pi / n)-\Delta_{\pi / n}^{k-1} g(t)\right\|_{\Phi}^{\prime} \\
& \leqq \inf \left\{j: c_{\Phi} \Phi(1 / i)\right. \\
& \left.\int_{0}^{2 \pi} \Phi\left(\Delta_{\pi / n}^{k-1} g(t+\pi / n)-\Delta_{\pi / n}^{k-1} g(t)\right) d t \leqq 1\right\} \\
& =\inf \left\{\mathrm{j}: c_{\Phi} \Phi(1 / j) \frac{1}{2 n} \int_{0}^{2 \pi} \sum_{k=1}^{2 n} \Phi\left(\Delta_{\pi / n}^{k-1} g\left(t+\frac{j \pi}{n}\right)\right.\right. \\
& \left.\left.-\Delta_{\pi / n}^{k-1} g\left(t+\frac{(j-1) \pi}{\mathrm{n}}\right)\right) d t \leqq 1\right\} \\
& \leqq \inf \left\{j: c_{\Phi} \Phi(1 / i) \frac{\pi}{n} \omega_{\Phi}(k ; g ; \pi / n) \leqq 1\right\} \\
& \leqq \inf \left\{j: \Phi(1 / j) \leqq \frac{n}{c_{\Phi} \pi \omega_{\Phi}(k ; g ; \pi / n)}\right\} .
\end{aligned}
$$

Thus 


$$
\begin{aligned}
\left\|\Delta_{\pi / n}^{k} g\right\|_{\Phi}^{\prime} & \leqq \frac{1}{\Phi^{-1}\left(\frac{n}{c_{\Phi} \pi \omega_{\Phi}(k ; g ; \pi / n)}\right)} \\
& \leqq \frac{1}{\Phi^{-1}(n) \Phi^{-1}\left(1 / c_{\Phi}{ }^{3} \pi\right) \Phi^{-1}\left(\frac{1}{\omega_{\Phi}(k ; g ; \pi / n)}\right)}
\end{aligned}
$$

and

$$
2^{k}\left|\alpha_{n}\right| \leqq \frac{1}{\Phi^{-1}\left(1 / c_{\Phi}^{3} \pi\right) \pi \Phi^{*}\left(\frac{1}{2 \pi}\right)} \cdot \frac{1}{\Phi^{-1}(n) \Phi^{-1}\left(\frac{1}{\omega_{\Phi}(k ; g ; \pi / n)}\right)}
$$

The inequality for $\left|\beta_{n}\right|$ is proved similarly.

Thus

$$
\begin{gathered}
\left.\max \left(\left|\alpha_{n}\right|\right),\left|\beta_{n}\right|\right) \leqq \\
\frac{2^{-k}}{\Phi^{-1}(n) \Phi^{-1}\left(\frac{1}{\omega_{\Phi}(k ; g ; \pi / n)}\right)} \\
\cdot \frac{1}{\Phi^{*}\left(\frac{1}{2} \pi\right) \Phi^{-1}\left(1 /\left(c_{\Phi}{ }^{3} \pi\right)\right) \pi} .
\end{gathered}
$$

Now, let $g=f^{(r)}, r \geqq 0$. Then

$$
k^{-r} \rho_{k}\left(f^{(r)}\right)=\rho_{k}(f)
$$

and if

$$
f(x) \sim \sum \alpha_{n} \cos n x+b_{n} \sin n x,
$$

then

$$
\begin{gathered}
\max \left(\left|a_{n}\right|,\left|b_{n}\right|\right) \leqq \\
2^{k-1} \Phi^{-1}(n) n^{r} \Phi^{-1}\left(\frac{1}{\omega_{\Phi}\left(k ; f^{(r)} ; \pi / n\right.}\right) \\
\cdot \frac{1}{\pi \Phi^{-1}\left(1 / c_{\Phi}^{3} \pi\right) \Phi^{*}\left(\frac{1}{2} \pi\right)}
\end{gathered}
$$

as desired.

The following class of functions was introduced by E. R. Love [5].

Definition. We say that a function $g$ is $\Phi$-absolutely continuous $(\Phi A C)$ if given $\epsilon>0$, there exists a $\delta_{0}>0$ such that 


$$
\sum \Phi\left(g\left(\beta_{i}\right)-g\left(\alpha_{i}\right)\right)<\epsilon
$$

holds for any nonoverlapping intervals $\left(\alpha_{i}, \beta_{i}\right)$ lying in the period and such that

$$
\sum \Phi\left(\beta_{i}-\alpha_{i}\right)<\delta_{0}
$$

THEOREM 2.8. If $f^{(r)} \in \Phi A C, \Phi a \Delta^{\prime} N$-function, then

$$
\rho_{n}(f)=o\left(\frac{1}{n^{r} \Phi^{-1}(n)}\right) .
$$

Proof. Select $\epsilon>0$. Then choose an integer $p$ such that $\Sigma \Phi(\pi / p)$ $=2 p \Phi(\pi / p)<2 p c \Phi(1 / p)<c \delta / \pi$, where $c \delta / \pi$ is the $\delta_{0}$ in the last definition, and so

$$
\left.\sum_{j=1}^{2 p} \Phi\left(f^{(r)}\right)\left(\frac{j \pi}{p}\right)-f^{(r)}\left(\frac{(j-1) \pi}{p}\right)\right)<\epsilon .
$$

Let $\left\{x_{i}\right\}$ be any partition of a period with mesh less than $1 / 2 p$ and group the elements $\Delta x_{i}=x_{i}-x_{i-1}$ so that

$$
\frac{1}{p}>\sum_{j_{1}}^{j_{2}} \Delta x_{i,} \geqq \frac{1}{2 p} \text {. }
$$

We have

$$
\sum \Phi\left(\Delta x_{i}\right)<\sum_{k} \Phi\left(\sum_{j=j_{k}}^{j_{k+1}} \Delta x_{i,}\right)<2 p \pi \Phi(1 / p)<\delta<\delta_{0},
$$

and therefore

$$
\sum \Phi\left(f^{(r)}\left(x_{i}\right)-f^{(r)}\left(x_{i-1}\right)\right)<\epsilon .
$$

Thus $V^{(1 /(2 p))}\left(f^{(r)}\right)<\epsilon$, hence

$$
V^{(\pi / n)}\left(f^{(r)}\right)=o(1) \text { as } n \rightarrow \infty .
$$

Using Lemma 2.7, with $k-1$,

$$
\rho_{n}(f)=\frac{1}{n^{r} \Phi^{-1}(n)} \mathrm{o}(1)=\mathrm{o}\left(\frac{1}{n^{r} \Phi^{-1}(n)}\right) .
$$

\section{REFERENCES}

1. N. Bari, Trigonometric Series I, II, Fizmatgiz, Moscow, 1961; English translation, Macmillan, New York.

2. E. Cohen, On The Degree Of Approximation Of A Function By The Partial Sums Of Its Fourier Series, Transactions of the AMS (to appear). 
3. B. I. Golubov, Continuous Functions Of Bounded P-variation, Math. Zametki, I (1967), 305-312, (Russian).

4. — On Functions Of Bounded P-variation, Math. USSR Izv. 2 (1968), $799-819$.

5. M. A. Krasnosel'skii and B. Rutickii, Convex Functions And Orlicz Spaces, English translation, P. Noordhoff Ltd., The Netherlands, 1961.

6. E. R. Love, A Generalization Of Absolute Continuity, J. London Math Society, 26 (1951), 1-13.

7. J. Musielak and W. Orlicz, On Generalized Variation (I), Studia Mathematica T. XVIII (1959), 11-41.

8. S. N. Nikol'skii, Fourier Series Of Functions Having Derivative Of Bounded Variation, Izv. Akad. Nauk SSR, Ser. Mat. 13 (1949), 513-532 (Russian).

9. N. Wiener, The Quadratic Variation Of A Function And Its Fourier Coefficients, J. Math and Phys. 3 (1924) 72-94.

10. L. C. Young, An Inequality Of The Hölder Type Connected With The Stielties Integration, Acta Mathematica 67 (1936), 251-282.

11. A. Zygmund, Trigonometrical Series I, II, rev. 2nd ed., Cambridge University Press, New York, 1968.

Department of Computer Science, University of Utah, Salt Lake City, Utah 84112 